\title{
Wird die Große Transformation geteilt? Zum Einfluss der Geschäftsmodelle von Sharing-Plattformen auf das transformative Potenzial der Sharing Economy
}

\author{
Nils S. Borchers \& Sebastian Jürss
}

Keywords: Sharing Economy, Transformation, Peer-to,Peer; Geschäftsmodell, Plattformkooperativismus

\section{Abstract}

Der Sharing Economy wird von ihren Befürworter*innen transformatives Potenzial zugeschrieben: Sie ermögliche ihren Nutzer*innen finanzielle Unabhängigkeit und mache das Wirtschaften nachhaltiger und demokratischer. Kritiker*innen sprechen ihr dieses Potenzial hingegen ab. Wir gehen in diesem Beitrag davon aus, dass die konkrete Ausgestaltung einer Sharing-Plattform dafür entscheidend ist, ob die Plattform - und dann in Summe: die Sharing Economy - in der Lage ist, einen transformativen Beitrag zu leisten oder nicht. Diese Ausgestaltung wiederum hängt vom jeweils adaptierten Geschäftsmodell ab. Wir befragen daher die sechs von Muñoz und Cohen (2017) vorgeschlagenen Dimensionen der Geschäftsmodelle von Sharing-Plattformen bezüglich ihrer Auswirkungen auf die transformativen Versprechen. Auf diese Weise können wir den Idealtyp einer transformativen Sharing-Plattform bestimmen. Die bekannten Sharing-Plattformen, insbesondere Airbnb und Uber, weichen jedoch deutlich von diesem Idealtyp ab. Ihnen sind daher Alternativen entgegenzusetzen, soll die Sharing Economy in den Dienst einer Großen Transformation gestellt werden.

Nils S. Borchers \& Sebastian Jürss: Wird die Große Transformation geteilt? Zum Einfluss der Geschäftsmodelle von Sharing-Plattformen auf das transformative Potenzial der Sharing Economy. In: Nils S. Borchers, Selma Güney, Uwe Krüger und Kerem Schamberger (Hrsg.): Transformation der Medien - Medien der Transformation. Verhandlungen des Netzwerks Kritische Kommunikationswissenschaft. Frankfurt am Main: Westend 2021. DOI: https://doi.org/10.53291/IPWR8205. 
Dr. Nils S. Borchers I Eberhard Karls Universität Tübingen I nils.borchers@ uni-tuebingen.de

Sebastian Jürss I Universität Bremen | s.juerss@uni-bremen.de

\section{Einführung}

Medientechnische Innovationen haben immer auch transformative Hoffnungen geweckt. Brecht (1982 [1932/33]) etwa beschrieb das transformative Potenzial des Radios als Empfangs- wie auch als Sendegerät, und Enzensberger (1997 [1970]) spekulierte auf einen emanzipativen Mediengebrauch durch die Demokratisierung der Videotechnik. Auch der epochemachende Medienumbruch, den wir in den vergangenen Jahrzehnten durch das Internet erleben, sorgte für weitreichende transformative Hoffnungen. In ihrem Kern heben diese Hoffnungen auf das Schaffen einer gerechteren Gesellschaft durch die Veränderung von Machtbalancen (sensu Elias 2009 [1970]) ab, die über unterschiedlichste Gesellschaftsbereiche hinweg in emanzipatorischen Vorstellungen ihren Ausdruck finden: in der Politik etwa in einer neu belebten partizipativen und deliberativen Demokratie (etwa Buchstein 1997), in der Wirtschaft im »Consumer Empowerment (etwa Labrecque et al. 2013) oder im Gesundheitsbereich im »Patient Empowerment« (etwa Borchers und Krömer 2015).

Besondere Hoffnungen wurden in diesem Zusammenhang in die Sharing Economy gesetzt, weil sie eine Medieninnovation auf die Organisation wirtschaftlicher Prozesse anwendet: Die Sharing Economy nutzt die Netzwerkstruktur des Internets, um neue Märkte zu schaffen (Chase 2015; Gansky 2010). Die Hoffnungen, die sich aus dieser Anwendung des Internets ergeben, verfestigen sich in drei Versprechen (Jürss und Borchers 2020): 1) ein nachhaltigerer Umgang mit begrenzten Ressourcen; 2) eine Demokratisierung der Wirtschaft; und 3) eine stärkere (finanzielle) Unabhängigkeit des Individuums. Diese Versprechen sind, wie noch zu zeigen sein wird, transformativ. Sie sind zudem anschlussfähig etwa zu den UN-Nachhaltigkeitszielen (United Nations o. J.), zu einer Großen (sozial-ökologischen) Transformation (Göpel 2020), durchaus auch im Rahmen eines ideologischen Programms wie dem Ökosozialismus (Kovel 2007). Nichtsdestotrotz ist die Sharing Economy verstärkt ins Kreuzfeuer der Kritik geraten (etwa Hill 2015; 
Scholz 2017). Denn offensichtlich war sie bislang nicht in der Lage, ihre Versprechen einzulösen.

Trotz der durchweg begründeten Kritik an der real existierenden Sharing Economy geht dieser Beitrag davon aus, dass die transformativen Hoffnungen, die in sie gesetzt wurden, prinzipiell berechtigt sind. Vor diesem Hintergrund fragen wir, wie eine Sharing Economy gestaltet sein müsste, um ihre transformativen Potenziale zu realisieren. Wir setzen dazu an den Sharing-Plattformen an, genauer noch: an ihren Geschäftsmodellen. Denn diese Plattformen stellen die Infrastruktur der Sharing Economy und bestimmen somit darüber, unter welchen Bedingungen ihre Nutzer*innen miteinander interagieren. Unser Ziel ist es, verschiedene Geschäftsmodelle hinsichtlich ihres Einflusses auf das Einlösen der transformativen Versprechen der Sharing Economy einzuschätzen.

\section{Die Sharing Economy als Akteurin einer sozial-ökologischen Transformation?}

\subsection{Zum Konzept der Sharing Economy}

Die Sharing Economy lässt sich - zumindest tentativ - bestimmen als »ein Set von Geschäftsmodellen, Plattformen und Austauschbeziehungen [...], in dem unter der Beteiligung von Privatpersonen (Peerto-Peer) Ressourcen, Dienstleistungen oder das Zugangsrecht zu Dingen (temporär) gegen eine Gebühr oder andere Kompensation [...] über Social-Media-Plattformen des Internets [...] verschenkt, getauscht, geliehen oder verkauft werden« (Hertwig und Papsdorf 2017, 524). Obwohl diese Definition eine gewisse konzeptionelle Klarheit suggeriert, ist die Sharing Economy kein eindeutig bestimmter Handlungsbereich. Keiner der vorgelegten Bestimmungsversuche (etwa Acquier, Daudigeos und Pinkse 2017; Frenken 2017; Hamari, Sjöklint und Ukkonen 2015; Muñoz und Cohen 2017) hat sich bislang etablieren können. Vor diesem Hintergrund ist es der Verdienst der hier angeführten Definition nach Hertwig und Papsdorf, einen Minimalkonsens innerhalb der Forschung bestimmen zu wollen. Dennoch dürften einige ihrer Definitionsentscheidungen strittig sein - etwa die Beschränkung auf Peer-to-Peer-Transaktionen oder die Festschreibung auf temporäre Aktivitäten, die eine dauerhafte Überlassung von 
Tauschobjekten ausschließt. Diese Uneinigkeit über die genaue Bestimmung der Sharing Economy hat unterschiedliche Gründe. Nicht unwesentlich dürfte sein, dass sich der Handlungsbereich zum einen noch immer als recht dynamisch erweist, so dass allzu enge Definitionen zum Teil nicht in der Lage sind, neue Entwicklungen zu erfassen. Zum anderen lassen sich aber auch politische Programme in der Definitionsfindung wiederfinden, die Einfluss darauf nehmen, wie eng oder weit das Konzept gefasst wird (zur Rekonstruktion der Ideologie der Sharing Economy siehe Jürss und Borchers 2020). Das Resultat: Sich voneinander deutlich unterscheidende Plattformen werden im Diskurs gemeinsam als Teile der Sharing Economy verhandelt. Wiederum Hertwig und Papsdorf (2017) differenzieren drei Segmente der Sharing Economy:

- die Tausch- und Schenkwirtschaft, in der güterspezifische Tauschbeziehungen bestehen, die weder Äquivalenz der getauschten Güter noch monetäre Kompensation vorsehen,

- die Nischen- und Nebenerwerbswirtschaft, in der Nutzer*innen vorrangig bislang nicht kommerzialisierte Dienstleistungen anbieten,

- die Plattformökonomie, in der für gewöhnlich Angebote und Dienstleistungen der bestehenden Ökonomie (der sogenannten »old economy«) unter Rekurs auf die Sharing-Rhetorik und mittels Plattformeinsatz auf digitale Märkte überführt und dort in der üblichen kapitalistischen Weise gehandelt werden.

Diese Unterschiedlichkeit der drei als Sharing Economy verhandelten Segmente spiegelt sich auch in der Beurteilung der Rolle wider, die die Sharing Economy in einer notwendigen gesellschaftlichen Transformation spielen kann.

\subsection{Die Versprechen der Sharing Economy und was daraus wurde}

Die Sharing Economy wurde von vielen Akteur*innen mit großer Euphorie begrüßt, nicht nur von Unternehmer*innen, sondern auch von Politiker*innen und Aktivist*innen. Diese Euphorie lässt sich nachvollziehen, nimmt man die Versprechen in den Blick, die der Sharing Economy zugeschrieben werden. Im Diskurs lassen sich drei zentrale Versprechen identifizieren (Jürss und Borchers 2020). 
Erstens soll die Sharing Economy eine effizientere Nutzung von Ressourcen und dadurch ein nachhaltiges Wirtschaften ermöglichen. Durch das Teilen von Bohrmaschinen, Zelten und Lebensmitteln über Sharing-Plattformen steigert sich deren - abstrakt gesprochen - Nutzungsintensität. So müssen weniger neue Bohrmaschinen, Zelte und Lebensmittel produziert werden. Das gelingt zum einen durch kurzfristiges Teilen in den Leerzeiten, während denen die Objekte von ihren Eigentümer*innen selbst nicht genutzt werden. So besagt die für die Sharing Economy ikonische Erzählung von der Bohrmaschine, dass diese im Schnitt nur zwischen 6 und 13 Minuten tatsächlich gebraucht wird und den Rest ihrer Existenz ungenutzt im Regal liegt (Botsman und Rogers 2011, 83). Sharing-Plattformen wie Peerby schaffen nun die Infrastruktur, um ungenutzte Geräte mit vertretbarem Aufwand zu verleihen. Zum anderen lässt sich die Nutzungsintensität steigern, indem man diese nach Bedarf verschenkt oder verkauft: Das nicht mehr benötigte Zelt wird dann über eBay veräußert.

Zweitens soll die Sharing Economy zu einer demokratischeren Form des Wirtschaftens führen. Weil (idealtypisch) Peers, also einander strukturell gleichgestellte Nutzer*innen, miteinander interagieren, werden Hierarchien abgeschafft. Gleichzeitig wird durch die Begegnung als Peers soziales Kapital generiert: Indem man über Couchsurfing bei privaten Gastgeber*innen übernachtet und nicht in einem Hotel, entstehen neue Bekannt- oder gar Freundschaften.

Drittens soll es die Sharing Economy ihren Nutzer*innen ermöglichen, (finanziell) unabhängig zu werden und sich so aus strukturellen Zwängen zu befreien. Sie schafft Sichtbarkeit für Privatpersonen, die als Anbietende auf einem Markt auftreten. Indem diese etwa ein leerstehendes Gästezimmer über Airbnb vermieten oder ihre Zeit samt Fähigkeiten einsetzen, um über TaskRabbit haushaltsnahe Dienstleistungen zu übernehmen, erschließen sich Plattformnutzer*innen als Mikro-Entrepreneure (Daum 2017) neue Einnahmequellen.

Diese Versprechen zeigen bereits an, wie die Sharing Economy transformativ wirken kann. ${ }^{1}$ Um es am Beispiel der UN-Nachhaltigkeitsziele (United Nations o.J.) nachzuzeichnen: Die Sharing Economy

1 Wenn wir in diesem Beitrag von Transformation sprechen, meinen wir eine gesellschaftlich wünschenswerte sozial-ökologische Transformation, wie sie an unterschiedlichen Stellen und mit unterschiedlichem politischem Unterbau imaginiert und propagiert wird, etwa in der Vorstellung einer »Großen Transformation« zur Nachhaltigkeit (Göpel 2020) oder im Konzept des Ökosozialismus (Kovel 2007), vgl. auch Krüger in diesem Band. 
unterstützt, so sie diese Versprechen erfüllt, insbesondere die Ziele der Nachhaltigkeit in Konsum und Produktion, der Verringerung von Ungleichheiten, der nachhaltigen Städte und Gemeinden sowie von menschenwürdiger Arbeit und Wirtschaftswachstum. Daneben bestehen zumindest indirekte Verbindungen zu einer Reihe weiterer UN-Ziele wie Maßnahmen zum Klimaschutz sowie zu Gesundheit und Wohlergehen. So wird die Sharing Economy potenziell zu einer Transformationsagentin.

Allerdings haben Kritiker*innen darauf hingewiesen, dass die Sharing Economy dieses Potenzial nicht nur nicht einlöst, sondern eine gesellschaftliche Transformation gar blockiert, indem sie die kapitalistische Ausbeutungslogik weiter forciert (etwa Hill 2015; Scholz 2017; Slee 2015). Dieser Einwand bezieht sich zumeist auf das Konzept des Plattformkapitalismus (Srnicek 2017) und blickt auf beobachtbare Dysfunktionalitäten wie die Wohnraumverknappung in touristischen Hotspots durch Airbnb-Vermietungen oder die geringen Einkommen und mangelnde soziale Absicherung der Gig-Worker von Uber. In den Augen der Kritiker*innen ist die Sharing Economy dann nicht weniger als »neoliberalism on steroids« (Murillo, Buckland und Val 2017).

Nun liegt es nahe, diese unterschiedlichen Positionen mit der Unterschiedlichkeit der als Sharing Economy verhandelten empirischen Phänomene in Verbindung zu bringen, wie sie Hertwig und Papsdorf (2017) beschreiben. An anderer Stelle haben wir nachgezeichnet, wie das Segment der Tausch- und Schenkwirtschaft in der Debatte dazu herhalten muss, um die Sharing Economy als Ganzes und damit auch das Segment der Plattformökonomie diskursiv zu legitimieren (Jürss und Borchers 2020). Hier stellen wir nun die Frage, wie eine Sharing Economy beschaffen sein müsste, um einen transformativen Beitrag zu leisten.

\section{Sharing-Plattformen und ihre Geschäftsmodelle}

Die einzelnen Sharing-Plattformen stellen die Infrastruktur der Sharing Economy dar. Sie sind es, die Märkte als Grundlage für Transaktionen schaffen. Darüber hinaus geben sie einseitig die Bedingungen vor, unter denen ihre Nutzer*innen als Teilnehmer*innen auf diesen Märkten miteinander interagieren, und bestimmen so deren Agency. Auch mit Blick auf die Sharing Economy gilt also Lessigs (2006) paradigmatische Feststellung »Code is law! « 
Die Sharing Economy kann mithin nur so transformativ sein, wie es die konkreten Plattformen zulassen. Deren Ausgestaltung hängt wesentlich von den Plattformbetreiber*innen ab, da diese sie zur optimalen Erreichung ihrer Ziele gestalten. Das verbindende Element zwischen diesen Zielen der Betreiber*innen und der Ausgestaltung der Plattform ist das Geschäftsmodell, auf dessen Grundlage Designentscheidungen getroffen werden (Abb. 1).

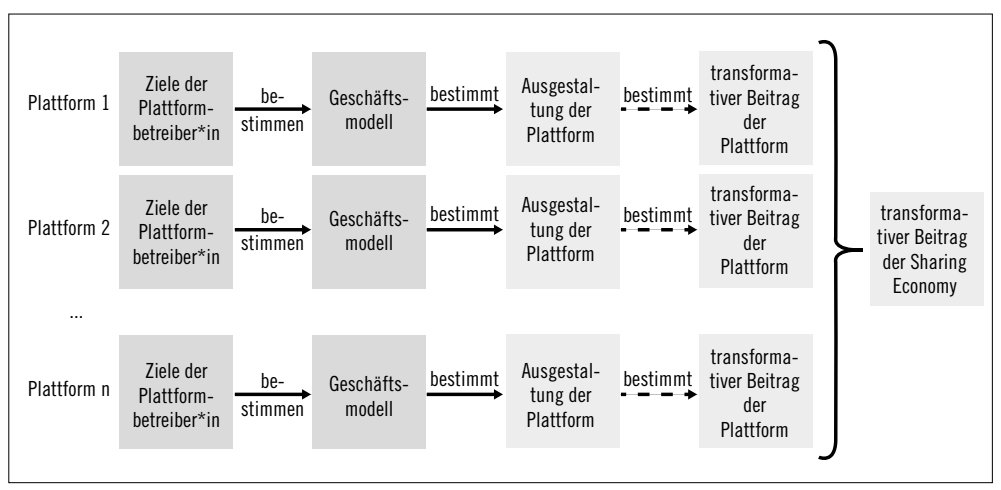

Abb. 1: Abhängigkeit des transformativen Beitrags der Sharing Economy vom Geschäftsmodell einzelner Sharing-Plattformen als Übersetzung der Ziele der Plattformbetreiber“innen.

Ein detailliertes Raster zur Analyse der Geschäftsmodelle von SharingPlattformen haben Muñoz und Cohen (2018) mit ihrem "Sharing business modell compass « vorgelegt. Dieser Kompass unterscheidet sechs Dimensionen: Plattform, Transaktion, Governance-Modell, Geschäftsansatz, Tauschobjekt und Technologie. Innerhalb dieser Dimensionen unterscheidet er jeweils drei Kategorien, in denen die einzelne Dimension ihre Ausprägung finden kann.

Bei der Erstellung des Kompasses gehen Muñoz und Cohen deduktiv vor, unter besonderem Einbezug der Forschungsergebnisse zur Sharing Economy. Ihre Überprüfung des Modells zeigt dann, dass der Kompass in der Lage ist, die Geschäftsmodelle von $36 \mathrm{zu}$ Testzwecken ausgewählten Plattformen zufriedenstellend abzubilden. Damit bietet er eine geeignete Grundlage für die Analyse in diesem Beitrag. 


\section{Die sechs Dimensionen der Geschäftsmodelle}

Im Folgenden werden wir für die sechs von Muñoz und Cohen (2018) vorgeschlagenen Dimensionen der Geschäftsmodelle von SharingPlattformen analysieren, welche Folgen ihre unterschiedlichen Ausprägungen auf den transformativen Beitrag der Sharing Economy zeitigen. Auf diese Weise möchten wir ermitteln, welche Ausprägungen zu wählen sind, damit eine Plattform die Versprechen der Sharing Economy einzulösen vermag. Zum Zweck der Komplexitätsreduktion werden wir für diese Prüfung vom »Optimalfall« ausgehen und eine kritische Masse an Nutzer*innen sowie kooperatives Verhalten voraussetzen.

\subsection{Plattform}

Muñoz und Cohen bestimmen den Typ der Plattform anhand einer strukturellen Verortung der Akteur*innen, die auf der Plattform als Anbietende und Nachfragende interagieren. Dazu unterscheiden sie zwischen institutionalisierten Akteur*innen (im Grunde: Unternehmen) und Privatpersonen. Erstere können in der Position der Anbietenden auftreten, Letztere sowohl in der Position der Anbietenden als auch der Nachfragenden. Daraus ergeben sich für den Plattformtyp drei mögliche Konstellationen: 1) Unternehmen als Anbietende und Nachfragende (in der Diktion von Muñoz und Cohen: business-tobusiness ( b2b); 2) Unternehmen als Anbietende, Privatpersonen als Nachfragende (business-to-crowd / b2c); und 3) Privatpersonen als Anbietende und Nachfragende (peer-to-peer / p2p). Tab. 1 zeigt, wie sich diese Ausprägungen auf die Versprechen der Sharing Economy auswirken.

Nach unserer Einschätzung besitzt der Plattformtyp eine entscheidende Bedeutung dafür, inwiefern die transformativen Versprechen eingelöst werden können. In der Diskussion der weiteren Dimensionen werden wir daher fragen, inwiefern bestimmte Ausprägungen nicht nur die drei eigentlichen Versprechen, sondern eben auch p2p-Transaktionen als entscheidenden Mechanismus einer transformativen Sharing Economy fördern (dazu auch Frenken 2017; Schor 2014).

Peer-to-Peer

Als Typ $p 2 p$ verhandeln Muñoz und Cohen Plattformen, bei denen nicht-institutionalisierte Akteur*innen sowohl in der Position der An- 
Tab. 1: Auswirkungen der Ausprägungen in der Dimension Plattform auf die Versprechen der Sharing Economy.

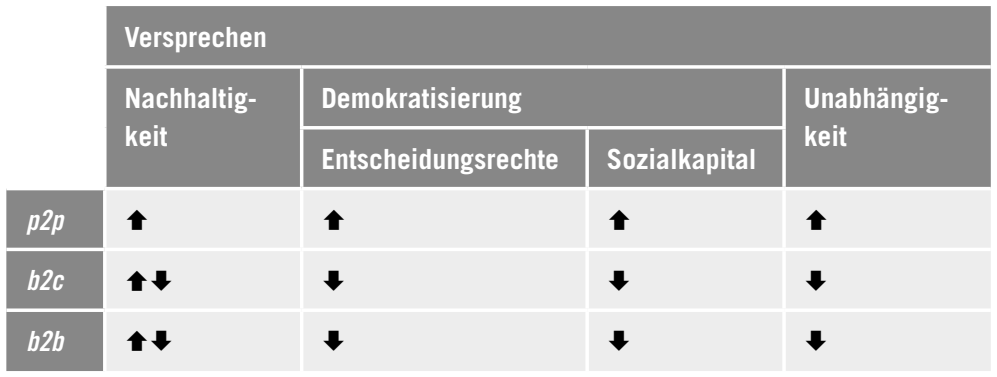

1 = positiver Einfluss der Ausprägung auf Versprechen; = kein positiver Einfluss (Festschreibung des Status quo) oder sogar negativer Einfluss; $\varnothing=$ kein Zusammenhang zwischen Ausprägung und Versprechen; = positiver oder negativer Einfluss, abhängig von Ausprägung in anderer Dimension; ( $)=$ positiver Einfluss, der aber hinter den Möglichkeiten zurückbleibt; p2p = Einfluss der Ausprägung auf das Entstehen von p2p-Konstellationen

bietenden als auch der Nachfragenden auftreten. Ein idealtypisches Beispiel für diesen Typ ist die Plattform Tauschgarten, die es Nutzer*innen ermöglicht, Pflanzensamen, Setzlinge und Ähnliches über Tauschtickets, die plattformeigene Währung (siehe Dimension Transaktion), untereinander zu handeln.

Um Muñoz und Cohens Differenzierung zu schärfen, sei an dieser Stelle angemerkt, dass die beiden Autoren im Diskurs üblicherweise p2p mit consumer-to-consumer-Interaktionen gleichsetzen. Diese Gleichsetzung wird deshalb vorgenommen, weil in der wirtschaftswissenschaftlichen Debatte Konsument*innen für gewöhnlich den Gegenpart zum Unternehmen darstellen. Als Konsument*innen werden sodann alle Akteur*innen kategorisiert, die nicht institutionalisiert sind. Allerdings wird hierbei unterschlagen, dass die Rolle des Peers voraussetzungsreicher ist als die der Konsument*in. Erstere erfordert nämlich, dass sich die Interaktionsteilnehmer*innen als strukturell gleichrangig begegnen und prinzipiell auch ihre Position wechseln können - also sowohl als Anbietende als auch als Nachfragende auftreten. Diese Erwartung wird dagegen nicht an Konsument*innen gerichtet. Unter diesem Gesichtspunkt fällt beispielsweise auf, dass die allgemeine Beschreibung etwa von Airbnb als einer p2p-Plattform nur bedingt zutrifft. Analytisch korrekt ist, dass dort Transaktionen zwischen Peers stattfinden, wenn etwa ein nicht genutztes Gästebett in einem Privathaushalt, in 
dem auch die Anbietenden leben, gebucht wird. Anders verhält es sich, wenn Anbietende eigens Wohnungen anmieten, um diese über Airbnb weiterzuvermieten.

In solchen Fällen mag die Anbietende zwar vordergründig als Privatperson (und damit als Konsument*in) auftreten. Analytisch handelt sie aber nicht mehr als Peer, weil sie durch die geschäftsmäßige Anmietung signalisiert, dass sie ausschließlich oder zumindest deutlich häufiger als Anbietende agiert - unabhängig davon, ob sie ihre Tätigkeit (ordnungsgemäß) als Gewerbe angemeldet hat und somit auch formal als institutionalisierte*r Akteur*in auftritt. Durch diese strukturelle Präferenz für die Position der Anbietenden wird nun aber die Reziprozitätserwartung außer Kraft gesetzt. Zu beobachten ist dann nichts anderes als die geschäftsmäßige Vermietung von Übernachtungsmöglichkeiten - eine Leistung, wie sie jedes Hotel erbringt. Entsprechend liegen in diesen Fällen analytisch keine p2p-, sondern b2c-Transaktionen vor, und Airbnb lässt sich daher mit dem Kategorisierungsraster von Muñoz und Cohen nicht eindeutig einem Plattformtyp zuordnen. Um diese Ungenauigkeit zu umgehen, werden wir in entsprechenden Fällen nicht auf Ebene der Plattformen, sondern der einzelnen Transaktionen argumentieren.

Sharing-Plattformen, die der Kategorie $p 2 p$ angehören, ermöglichen es Privatpersonen, mit ihren Angeboten auf dem Markt auffindbar zu werden. Natürlich konnten Hobbygärtner*innen auch zuvor versuchen, Pflanzensamen aus ihrem Garten in kleinen Mengen gegen Samen Gleichgesinnter zu tauschen. Der Aufwand, sich als Anbietende zu identifizieren, liegt ohne entsprechende Plattformen allerdings deutlich höher. Möglichkeiten sind hier etwa das Herumfragen im Bekanntenkreis oder der Aushang an einem Schwarzen Brett - Möglichkeiten, die eine deutliche lokale und temporale, im ersten Fall zudem noch eine deutliche soziale Beschränkung aufweisen. Spezialisierte Zeitschriften, die Kleinanzeigen aufnehmen, sind zwar lediglich temporal beschränkt, dafür kostet das Inserat jedoch in der Regel Geld. Entsprechend muss auch in diesem Fall von hohen Transaktionskosten - also den nötigen Ressourcen, damit eine Transaktion vonstattengehen kann - ausgegangen werden. Diese Kosten können gerade bei Tauschobjekten, denen Transaktionspartner*innen einen verhältnismäßig geringen Wert zuschreiben, stark ins Gewicht fallen, so dass sie davon absehen könnten, ihre Leerlaufkapazitäten (»idle resources«) feilzubieten beziehungsweise sich auf die Suche nach entsprechenden Tauschobjekten zu begeben. Im Vergleich dazu ermöglichen Sharing-Plattformen eine entscheidende Senkung der anfallenden Kosten. Sie heben temporale, 
lokale sowie soziale Beschränkungen auf und machen Angebote ebenso unaufwändig (Transaktionskosten) wie erwartbar (Erfolgswahrscheinlichkeit) auffindbar.

Aus dem vereinfachten Marktzugang folgt hinsichtlich des Versprechens auf Unabhängigkeit, dass p2p-basierte Plattformen ihre Nutzer*innen in die Lage versetzen, neue Einkommensquellen zu erschließen, indem sie ihre Leerlaufkapazitäten zur Nutzung anbieten. Das beeinflusst wiederum das Nachhaltigkeitsversprechen: Nur wenn es sich für Privatpersonen lohnt (sei es finanziell oder ideologisch), ihre Leerlaufkapazitäten als Tauschobjekte anzubieten, können Nachfragende auf diese zugreifen, statt neu produzierte Objekte zu erwerben. So wird die steigende Nachfrage nach Übernachtungsmöglichkeiten durch die Bereitstellung freier Betten in einzelnen Privathaushalten befriedigt, statt neue Hotelkapazitäten zu schaffen. Positive Effekte von $\mathrm{p} 2 \mathrm{p}$-Transaktionen sind aber auch hinsichtlich des Versprechens auf Demokratisierung zu erwarten. Denn die Reziprozitätserwartung, der zufolge Nutzer*innen sowohl in der Angebots- als auch der Nachfrageposition agieren sollten, führt dazu, dass sich Peers prinzipiell als strukturell Gleiche begegnen. Dafür sorgt nicht zuletzt die Tatsache, dass sie durch den Rollenwechsel Empathie für die jeweils andere in ihrer Rolle ausbilden, denn ihnen sind die Bedingungen auch deren Rolle bekannt. Schließlich ist zu erwarten, dass p2p-Transaktionen die Bildung von Sozialkapital fördern, da sie die Möglichkeit der Begegnung ebenbürtiger Partner*innen stiften. Es ist zwar zu vermuten, dass der Pathos, mit dem dieser Aspekt von so mancher Sharing-Plattform eingebracht wird, eine Übertreibung darstellt, weil Begegnungen von Peers in diesem Kontext immer auch zweckfunktional sein können und müssen: Man trifft sich (etwa über Paul Camper), um den Wohnmobilschlüssel zu übergeben, und macht genau das. Nichtsdestotrotz verweist die Peer-Konstellation durch die strukturelle Gleichheit auf die Person hinter der Rolle: Man wird eher mit einem Peer-Anbietenden auch über die Erlebnisse des Tages oder die bevorstehende Reise plaudern als mit der Angestellten der Automobilvermietung, die auf die Rolle der Unternehmensvertreterin festgeschrieben ist. So berichten etwa Nutzer*innen von Plattformen wie Couchsurfing davon, dass durch die dort vermittelten Transaktionen Bekannt- oder gar Freundschaften entstanden sind (Decrop et al. 2018).

Damit handelt es sich bei der Kategorie $p 2 p$ um die einzige Kategorie (über alle Dimensionen hinweg), die sich durchweg positiv auf die Erfüllung der drei Versprechen auswirkt. 


\section{Business-to-Crowd}

Unter den Typ $b 2 c$ fallen nach Muñoz und Cohen Plattformen, die die Position der Anbietenden auf institutionelle Akteur*innen und die der Nachfragenden auf nicht-institutionalisierte Akteur*innen festschreiben. Ein Beispiel ist die aus dem Zusammenschluss von car2go (Daimler) und DriveNow (BMW) entstandene Plattform Share Now, die (ausschließlich) firmeneigene Fahrzeuge zur Kurzmiete anbietet. Im Grunde handelt es sich dabei also um eine gewöhnliche Autovermietung, die ihre Transaktionen lediglich über eine eigens dafür aufgesetzte Plattform verwaltet.

Mit Blick auf das Versprechen der Nachhaltigkeit lässt sich für b2cPlattformen feststellen, dass die zur Verfügung gestellten Tauschobjekte dem Markt in der Regel eigens zum Zweck der kommerziellen Nutzung zugeführt werden, um über ihre Vermietung Profit zu erwirtschaften (siehe auch Dimension Tauschobjekt). Share Now hat beispielsweise die dort buchbaren Fahrzeuge speziell als Neuwagen zugelassen, um Geld an ihnen zu verdienen. Analog mieten kommerzielle Vermieter*innen auf Airbnb Wohnungen eigens zu dem Zweck an, diese ihrerseits weiterzuvermieten, und entziehen sie so dem Mietmarkt. Das impliziert, dass hier eben nicht Leerlaufkapazitäten effizienter genutzt, sondern für die Angebotserstellung neue Produkte hergestellt werden - sei es unmittelbar (Neubau von Automobilen bei Share Now) oder mittelbar (Neubau von Wohnungen, um die dem Markt entzogenen Wohnungen zu ersetzen bei Airbnb). Entsprechend zeigen Daten für Berlin und Hamburg, dass der Ausbau von kommerziellen Carsharing-Angeboten nicht zu einer Verringerung der Autozulassungen geführt hat (Kearney, o. J.). Auch das Versprechen der Unabhängigkeit kann von Plattformen des Typs $b 2 c$ nicht erfüllt werden, weil sie per definitionem einzig institutionelle Akteur*innen in der Position der Anbietenden zulassen. Diese Exklusivität hat außerdem zur Folge, dass sich über entsprechende Plattformen keine Peers begegnen. Zu beobachten sind also Geschäftsbeziehungen zwischen Unternehmen und Kund*innen, die sich strukturell nicht von den hinlänglich bekannten Geschäftsbeziehungen der »old economy« unterscheiden. Folglich kann der b2c-Typ auch das Demokratisierungsversprechen nicht einlösen.

\section{Business-to-Business}

b2b-Plattformen sind Plattformen, auf denen Unternehmen sowohl in der Angebots- als auch der Nachfrageposition auftreten. Als Beispiel nennen Muñoz und Cohen das Logistikunternehmen Cargomatic, das 
freie Ladekapazitäten von Lastkraftwagen vermittelt. ${ }^{2}$ Auf diese Weise werden tatsächlich ungenutzte Ressourcen effizienter eingesetzt, so dass entsprechende b2b-Plattformen den Ressourcenverbrauch verringern können. Dies gilt natürlich nur dann, wenn wie bei Cargomatic bereits bestehende Kapazitäten zum Einsatz kommen, und nicht wie bei Share Now eigens hergestellt werden, um sie über die Plattform anzubieten (siehe Dimension Tauschobjekt). Für die Versprechen Unabhängigkeit und Demokratisierung gilt hingegen das, was wir im vorangegangenen Abschnitt bereits für b2c-Plattformen festgestellt haben: Durch die Festschreibung der Position der Anbietenden auf institutionelle Akteur*innen tragen b2b-Plattfromen nicht zur Erfüllung dieser Versprechen bei.

\subsection{Transaktion}

Transaktionstypen lassen sich nach Muñoz und Cohen $(2018,131)$ anhand des Ausmaßes unterscheiden, sto which transactions on the platform are left to market forces or if they are suppressed or altered by the intermediary". Im Prinzip verbirgt sich dahinter die Frage, ob - und wenn ja: wie - Transaktionen kompensiert werden. Der Kompass unterscheidet drei Typen von Transaktionen: Markt, Alternativ und Frei. Tab. 2 gibt einen Überblick über ihre jeweiligen Auswirkungen.

\section{Markt}

Der Typ Markt ist dadurch charakterisiert, dass Transaktionen mit Geld kompensiert werden, also mit einer Währung, die auch außerhalb der Plattform genutzt werden kann. Dadurch, dass das Angebot einen Wert erhält, der in einer allgemeingültigen Währung angegeben ist, so argumentieren Muñoz und Cohen, hält »der Markt« Einzug in die Transaktion. Denn die Preisgestaltung der Anbietenden auf der Plattform sollte, der Logik der Preisabhängigkeit von Angebot und Nachfrage folgend,

2 Zwei weitere von Muñoz und Cohen als Beispiel angeführte Plattformen, Cohealo und Munirent, ermöglichen die Organisation des Ressourceneinsatzes zwischen Abteilungen eines einzigen (Groß-)Unternehmens. Hierbei handelt es sich aber um ein organisationsinternes Wissensmanagementsystem, weshalb die beiden Plattformen nicht als Marktplatz kategorisierbar sind, weil ein und derselbe Akteur als Anbieter und als Nachfragender auftritt. 
Tab. 2: Auswirkungen der Ausprägungen in der Dimension Transaktion auf die Versprechen der Sharing Economy (Legende siehe Tab. 1).

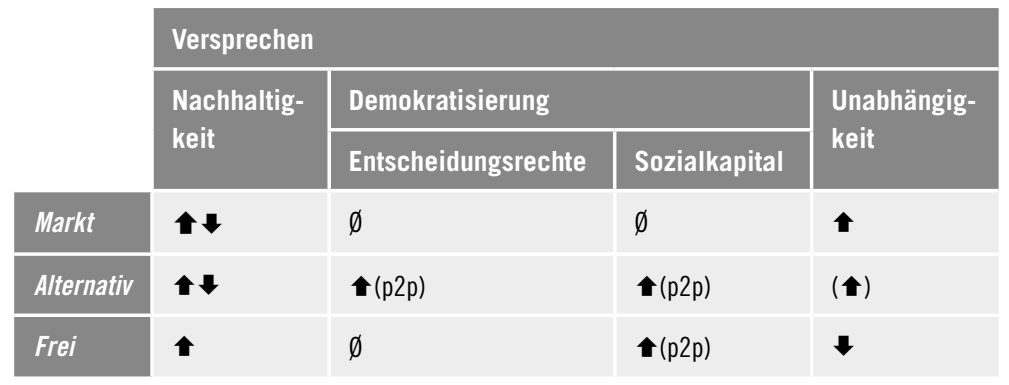

die Preise von vergleichbaren Angeboten außerhalb der Plattform (also etwa des Zimmers in besagtem Hotel) berücksichtigen.

Die Einnahmen können Anbietende beim Transaktionstyp Markt aufgrund der allgemeinen Gültigkeit der Währung vielfältig einsetzen (Lebensmittel einkaufen, an Sea Watch spenden und Ähnliches). Auf diese Weise eröffnen Plattformen dieses Transaktionstyps neue, potenziell substantielle Einkommensquellen. Mit Blick auf das Unabhängigkeitsversprechen lässt sich also konstatieren, dass die Nutzung allgemeingültiger Währungen seine Einlösung unterstützt. Gleichzeitig schafft sie die Möglichkeit, frei verwendbare Einkommen zu generieren, Anreize für das Upscaling, also eine Steigerung der Transaktionsanzahl. Solche Upscaling-Prozesse sind mit Blick auf das Unabhängigkeitsversprechen positiv zu bewerten. Auch das Versprechen der Nachhaltigkeit profitiert, wenn die Anreize potenzielle Anbieter dazu motivieren, ihre Leerlaufkapazitäten tatsächlich anzubieten. Am Beispiel: Lässt sich mit der Vermietung der eigenen Bohrmaschine Geld verdienen, lohnt sich der damit verbundene Aufwand. So muss die Nachfrage nicht durch neu produzierte Bohrmaschinen befriedigt werden.

Der Transaktionstyp Markt hat aber auch negative Aspekte: Zum einen können die Anreize dazu führen, dass Anbieter statt Leerlaufkapazitäten neue oder bislang anders genutzte Ressourcen über die Plattform anbieten: Wohnungen werden dem Mietmarkt entzogen, um sie über Airbnb in Kurzzeitvermietung rentabler zu vermieten - was unter Umständen die Mietpreise in die Höhe treibt (Unabhängigkeit) und lokale Sozialstrukturen zerstört (Demokratisierung). Auch können ein gesteigertes Angebot an Übernachtungsmöglichkeiten und möglicherweise sinkende Übernachtungspreise zu einem Anstieg an Reisen füh- 
ren (Nachhaltigkeit). Zum anderen ist eine Kommodifizierung bislang kostenloser Produkte und Leistungen denkbar - wenn beispielsweise die nachbarschaftliche Einkaufshilfe nunmehr mit Hilfe von SharingPlattformen in Wert gesetzt wird.

Der Transaktionstyp Markt ermöglicht es Plattformnutzer*innen zudem, einseitig in einer Position der Anbietenden oder der Nachfragenden auf der Plattform zu agieren. Er erzwingt damit keine p2pStruktur, wie es beim folgenden Transaktionstyp Alternativ der Fall ist, weshalb entsprechende Plattformen auch nicht in der Lage sind, qua Design p2p-Effekte zu provozieren (siehe Dimension Plattformtyp).

\section{Alternativ}

Es gibt Plattformen, die eigene Währungen eingeführt haben, wie beispielsweise die sogenannten TimeCoins auf TimeRepublik. Muñoz und Cohen ordnen diese Fälle dem alternativen Transaktionstyp zu. Die plattformeigenen Währungen werden nur dort als Zahlungsmittel akzeptiert und besitzen keinen darüberhinausgehenden Wert, was die Anwendungsmöglichkeiten stark einschränkt. Die Nicht-Übertragbarkeit des auf der Plattform generierten Einkommens in andere Handlungszusammenhänge begrenzt das Upscaling und damit die Möglichkeit für Nutzer*innen, sich neue, substanzielle Einkommensquellen zu erschließen. Um das Versprechen auf Unabhängigkeit zu erfüllen, ist der alternative Transaktionstyp also nur bedingt geeignet. Allerdings sorgt die Nicht-Übertragbarkeit der plattformeigenen Währung auch dafür, dass der über die Plattform erwirtschaftete Mehrwert in der Community verbleibt. Es gibt schlicht keine Abflussmöglichkeiten, so dass Nutzer*innen das Einkommen nur dort einsetzen können. Diese Abflusssperre stärkt die p2p-Struktur, weil es den Rollenwechsel qua Design forciert: Das Erwirtschaften eines Einkommens in der Position der Anbietenden ist für Nutzer*innen nur dann sinnvoll, wenn dieses zu einem anderen Zeitpunkt in der Position der Nachfragenden auch genutzt wird. Mit Blick auf das Versprechen der Nachhaltigkeit ist die Bewertung des alternativen Transaktionstypen nicht eindeutig.

\section{Frei}

Als freie Transaktionen bezeichnen Muñoz und Cohen solche, in denen Anbietende Angebote erstellen, ohne dafür eine Gegenleistung zu verlangen. Dieser Typ findet sich etwa auf den Plattformen Couchsurfing (Übernachtungsmöglichkeit) und foodsharing (Lebensmittel). Entsprechende Plattformen werden daher mitunter als Teil einer Schenk- 
wirtschaft (»gift economy«) verhandelt (Mikołajewska-Zając 2016). Bei diesem Transaktionstyp ist ein Upscaling nicht sinnvoll. Wenn die Anbietenden keine Gegenleistungen verlangen können, lohnt sich der Ausbau der Aktivitäten nicht. Damit bietet dieser Plattformtyp auch keine Möglichkeiten, alternative Einkommensquellen zu erschließen. Aus Perspektive der Nachfragenden ermöglicht die Nutzung hingegen eine Reduktion allgemeiner Ausgaben, da sie die benötigten Produkte kostenfrei erhalten und diese nicht auf einem Markt erwerben müssen (etwa das Abendessen aus der foodsharing-Box). Mit Blick auf das Nachhaltigkeitsversprechen lässt sich zudem festhalten, dass der Transaktionstyp Frei durch Senkung der Transaktionskosten dazu beitragen kann, Leerlaufkapazitäten zu nutzen: Das Essen wird aufwandsniedrig gespendet, statt es wegzuwerfen. Auf diese Weise zahlen Plattformen, die den freien Transaktionstyp nutzen, auf das Versprechen der Nachhaltigkeit ein.

Wie schon der Transaktionstyp Markt bietet auch der freie Transaktionstyp die Option, die Plattform einseitig in einer Rolle zu nutzen. Positionswechsel zwischen Anbietenden zu Nachfragenden werden nicht qua Design forciert. Nichtsdestotrotz ist zumindest bei einigen Plattformen eine konkrete Reziprozitätserwartung zu beobachten: Wer über Couchsurfing bei anderen Nutzer*innen nächtigt, sollte auch selbst Gäste beherbergen (Liu et al. 2016). Plattformen können allerdings auch ohne eine solche Reziprozitätserwartung operieren, da der übergreifende Transaktionstyp Frei ihr Vorhandensein nicht erzwingt. Wie stark diese Reziprozitätserwartung ist, hängt maßgeblich von der durch die Plattformbetreiber*innen formulierte Vision sowie von der gelebten Praxis in der Plattform-Community ab.

\subsection{Governance-Modell}

Muñoz und Cohen unterscheiden die Geschäftsmodelle von Plattformen in der Dimension Governance-Modell danach, wie Entscheidungen getroffen und der Werttausch (»value exchange«) organisiert werden. Die drei Ausprägungen sind: Korporativ, Kollaborativ und Kooperativ. Mit Blick auf die aktuelle Diskussion um Plattformkooperativen drängt es sich jedoch auf, zwei Varianten des kooperativen Typs danach zu differenzieren, ob lediglich die Anbietenden oder alle Nutzer*innen an diesen beteiligt sind. Tab. 3 liefert einen ersten Überblick über die Auswirkungen. 
Tab. 3: Auswirkungen der Ausprägungen in der Dimension Governance-Modell auf die Versprechen der Sharing Economy (Legende siehe Tab. 1).

\begin{tabular}{|c|c|c|c|c|}
\hline & \multicolumn{4}{|c|}{ Versprechen } \\
\hline & \multirow{2}{*}{$\begin{array}{l}\text { Nachhal- } \\
\text { tigkeit }\end{array}$} & \multicolumn{2}{|l|}{ Demokratisierung } & \multirow{2}{*}{$\begin{array}{l}\text { Unabhän- } \\
\text { gigkeit }\end{array}$} \\
\hline & & Entscheidungsrechte & Sozialkapital & \\
\hline Korporativ & $\emptyset$ & $\downarrow$ & $\emptyset$ & 十七 \\
\hline Kollaborativ & $\emptyset$ & $\downarrow$ & $\emptyset$ & คt \\
\hline Kooperativ (V1) & $\emptyset$ & $(\mathbf{+})$ & $(p 2 p)$ & - \\
\hline Kooperativ (V2) & - (p2p) & - & - (p2p) & - \\
\hline
\end{tabular}

\section{Korporativ}

Zum korporativen Typ gehören laut Muñoz und Cohen (2018, 133) Plattformen, die »traditional corporate governance models« adaptieren. De facto sind damit diejenigen Plattformen gemeint, die wie ein herkömmliches Unternehmen hierarchisch geführt werden. Die Autoren weisen selbst darauf hin, dass die Mehrzahl der Sharing-Plattformen in diese Kategorie fällt - darunter der Großteil der bekannteren Vertreter wie Airbnb, Uber, BlaBlaCar, TaskRabbit oder Share Now. Bei diesem Governance-Modell sind die Nutzer*innen nicht in die Entscheidungsfindung eingebunden. Stattdessen werden diese durch das Management, gegebenenfalls in Abstimmung mit den Eigentümer*innen, getroffen. Entsprechend tragen Plattformen dieses GovernanceModells nicht zur Demokratisierung bei. Mit Blick auf das Versprechen der Unabhängigkeit ist es generell möglich, dass Nutzer*innen neue Einkommensquellen erschließen, wenn die Plattform einen entsprechenden Transaktionsmodus verwendet (siehe Dimension Transaktion). Es liegt allerdings für die Betreiber*innen nahe, einen Geschäftsansatz zu wählen, der auf das Erwirtschaften von Profiten abzielt (profitgetrieben oder hybrid). Daher gelten hier die in diesem Zusammenhang bereits diskutierten Limitationen (siehe Dimension Geschäftsansatz).

\section{Kollaborativ}

Die Informationen, die Muñoz und Cohen zum kollaborativen Governance-Modell bereitstellen, sind recht begrenzt. Sie bemerken lediglich 
im Kontext einer Plattform zum Mieten und Vermieten von Haushaltsgegenständen: »Peerby raised a funding round via crowdfunding with their own user community, which we consider to be reflective of collaborative governance, because the user community became partial coowners in the platform as a result." (Muñoz und Cohen 2018, 133) Anders als im Zitat angedeutet, war die Finanzierungsrunde von Peerby allerdings nicht auf Nutzer*innen der Plattform beschränkt, sondern für alle interessierten Investor*innen offen. Folglich unterscheidet sich die Bewertung des kollaborative Governance-Typs damit nicht vom korporativen: Ähnlich wie man sich (das nötige Investitionskapital und die entsprechende Gesinnung vorausgesetzt) Aktien von Uber zulegen kann, ist es auch möglich, sich über die Crowfunding-Aktion als Shareholder bei Peerby einzukaufen, freilich ohne dass damit eine Stimmberechtigung einherginge. Hinsichtlich der Versprechen besitzt die Bewertung des korporativen Typs demnach Gültigkeit auch für den kollaborativen Typ.

\section{Kooperativ}

Dem kooperativen Typ ordnen Muñoz und Cohen Plattformen zu, die genossenschaftlich organisiert sind. Hier suchen sie also Anschluss an die Debatte zum Plattformkooperativismus (Scholz und Schneider 2017). Genossenschaften zeichnen sich gemeinhin dadurch aus, dass die Mitarbeiter*innen gleichzeitig Eigentümer*innen des Unternehmens sind. Im Kontext der Sharing Economy wird diese Idee dann zumeist auf die Nutzer*innen der Sharing-Plattform bezogen. Hier sind in der Regel zwei Varianten zu unterscheiden, die von Cohen und Muñoz selbst nicht weiter differenziert werden.

In Variante 1 treten nur die Anbietenden als Eigentümer*innen auf. Als Beispiel können kooperative Zusammenschlüsse von Taxifahrer*innen dienen, die gemeinsam eine Plattform betreiben, um Fahrten zu vermitteln (etwa Green Taxi Cooperative). Diese Variante, die auch Muñoz und Cohen maßgeblich vor Augen schwebt, hat vor allem Scholz (2017) in Abgrenzung zu Uber ausgearbeitet. Prüft man ihre Auswirkungen auf die Versprechen der Sharing Economy, stellt man zunächst fest, dass sie - in Verbindung mit einem entsprechenden Transaktionstyp - das Erschließen neuer Einkommensquellen ermöglicht. Verglichen mit den beiden bereits besprochenen Governance-Typen ist ihr Potenzial hierfür sogar größer: Die Tatsache, dass die Anbietenden gleichzeitig die Eigentümer*innen sind, ermöglicht es, die Gebühren für die Abwicklung von Transaktionen zu minimieren. Es gibt schließ- 
lich nur eine Akteur*innengruppe, die an den Transaktionen verdienen möchte - und nicht zwei wie bei der Trennung von Anbietenden und Eigentümer*innen. Im Vergleich zu den beiden anderen GovernanceTypen lässt sich sodann konstatieren, dass Variante 1 der kooperativen Plattformen zum Versprechen der Demokratisierung beiträgt, weil Anbietenden als Eigentümer*innen ein Mitspracherecht bei Entscheidungen eingeräumt wird. Die Entstehung von Sozialkapital wird durch diese Variante allerdings nicht gefördert, weil sie dazu neigt, Plattformnutzer*innen auf nur eine Position - Anbietende oder Nachfragende festzuschreiben. Da erstere gleichzeitig die Eigentümer*innen der Plattform sind, muss man zur Eigentümer*in werden, um die Rolle der Anbietenden einzunehmen. Der Rollenwechsel wird dadurch deutlich erschwert, so dass im Fall von Variante 1 p2p-Interaktionen unterdrückt werden. Entsprechend handelt es sich bei den Eigentümer*innen (und Anbietenden) des Green Taxi Cooperative in der Regel um hauptberufliche Taxifahrer*innen.

Variante 2 des kooperativen Typs löst diese Festschreibung auf, indem sie alle Nutzer*innen der Plattform zu Mitgliedern der Plattform-Kooperativen und damit zu Eigentümer*innen macht. Für diese Variante steht vor allem die Idee des »Open Cooperativism«, die das Konzept der Kooperativen mit dem der »Commons-based Peer Production «, also der gemeinsamen Produktion in einer als Allmende organisierten Gemeinschaft, zusammendenkt (Bauwens und Kostakis 2014; Pazaitis, Kostakis und Bauwens 2017). Auf diese Weise sind zum einen p2p-Interaktionen möglich, zum anderen wird das Versprechen auf Demokratisierung umfassender erfüllt, indem nun alle Nutzer*innen und nicht mehr nur die Anbietenden ein Mitspracherecht erhalten.

\subsection{Geschäftsansatz}

Die Dimension Geschäftsansatz erfasst das Ziel, mit dem eine Plattform betrieben wird. Muñoz und Cohen unterscheiden finanzielle von ideellen Absichten. Daraus ergibt sich eine Kategorisierung in profitgetriebene und missionsgetriebene Geschäftsansätze sowie hybride Mischformen. Tab. 4 bietet einen Überblick über die Auswirkungen der drei Varianten. 
Tab. 4: Auswirkungen der Ausprägungen in der Dimension Geschäftsansatz auf die Versprechen der Sharing Economy (Legende siehe Tab. 1).

\begin{tabular}{|c|c|c|c|c|}
\hline & \multicolumn{4}{|c|}{ Versprechen } \\
\hline & \multirow{2}{*}{$\begin{array}{l}\text { Nachhal- } \\
\text { tigkeit }\end{array}$} & \multicolumn{2}{|l|}{ Demokratisierung } & \multirow{2}{*}{$\begin{array}{l}\text { Unabhän- } \\
\text { gigkeit }\end{array}$} \\
\hline & & Entscheidungsrechte & Sozialkapital & \\
\hline Profitgetrieben & ค & $\emptyset$ & $\emptyset$ & (1) \\
\hline Missionsgetrieben & $\emptyset$ & $\emptyset$ & $\boldsymbol{1}$ & - \\
\hline Hybrid & At & $\emptyset$ & $\emptyset$ & 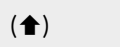 \\
\hline
\end{tabular}

\section{Profitgetrieben}

Plattformen fallen in die Kategorie Profitgetrieben, wenn ihr Ziel in der Erwirtschaftung eines Profits für die Eigentümer*innen besteht. Ein Großteil der bekannten Anbieter gehört zu dieser Kategorie, beispielsweise Uber, Airbnb oder Helpling. Plattformen aus dieser Kategorie können generell dazu beitragen, dass die Sharing Economy ihr Versprechen auf Nachhaltigkeit einlöst, etwa indem das leerstehende Gästezimmer über Airbnb vermietet wird. Allerdings setzt das Ziel, Profit zu erwirtschaften, einen Anreiz dafür, die Plattform so zu gestalten, dass auch neu produzierte Tauschobjekte und nicht allein Leerlaufkapazitäten auf der Plattform getauscht werden - wie im Falle des Ankaufs eines Wohnwagens zur Vermietung über Paul Camper.

Zusätzliche Angebote erhöhen die Chance zusätzlicher Transaktionen und damit eines höheren Profits. Werden Plattformen auf diese Weise gestaltet, können sie sich negativ auf das Versprechen der Nachhaltigkeit auswirken (siehe Dimension Tauschobjekt). Die Einlösung des Unabhängigkeitsversprechens scheint dagegen möglich: Sobald die Transaktionsgebühr eine Einnahmequelle der Plattformbetreiber*innen darstellt, werden sie daran interessiert sein, dass für Transaktionen gezahlt wird (Transaktionstyp Markt). Binden sie deren Höhe an den jeweiligen Preis, werden sie zudem daran interessiert sein, Mechanismen einzuführen, die zur Maximierung der abrufbaren Preise beitragen. Von diesen Anreizen für die Plattformbetreiber*innen profitieren auch die Anbietenden. Allerdings werden Plattformbetreiber*innen ebenfalls daran interessiert sein, die Transaktionsgebühr zu maximieren. Damit führt das Ziel der Profitgenerierung aus Perspektive der Anbietenden dazu, 
dass sie einen nicht unerheblichen, von den Plattformbetreiber*innen maximierten Teil des erwirtschafteten Mehrwerts als Gebühr abführen müssen. Sie können also eine neue Einkommensquelle erschließen, ihr Erlös ist wegen der Gebühr jedoch geringer, als er sein könnte.

\section{Missionsgetrieben}

Muñoz und Cohen stellen profitgetriebenen Plattformen missionsgetriebene gegenüber, die sich auf ein allgemeinnütziges Ziel verpflichten. Unter diese Kategorie fällt beispielsweise foodsharing, über das die Anbietenden Lebensmittel, die sie nicht mehr benötigen, an andere Nutzer*innen abgeben können.

Weil missionsgetriebene Plattformen keinen Profit erwirtschaften müssen, können sie auf eine Transaktionsgebühr verzichten. Somit unterstützen sie in Kombination mit den Transaktionstypen Alternativ und insbesondere Markt das Versprechen auf Unabhängigkeit besser als profitgetriebene Plattformen, die in der Regel einen Teil des Mehrwerts per Gebühr abschöpfen. Die Verpflichtung der missionsgetriebenen Plattformen auf ein gemeinnütziges Ziel zieht auch Nutzer*innen an, die ähnliche Werte vertreten. Dadurch begegnen sich in diesem Kontext Gleichgesinnte, was wiederum die Wahrscheinlichkeit der Entstehung von Sozialkapital erhöht, weil das gemeinsame Anliegen als Grundlage für ein Kennenlernen dienen kann.

\section{Hybrid}

Ansätze, die sowohl profit- als auch missionsgetrieben sind, bezeichnen Muñoz und Cohen als Hybriden. Sie haben hierbei vor allem (USamerikanische) B Corporations wie Kickstarter oder ehemals Etsy im Blick - also For-Profit-Unternehmen, die sich ihr (zusätzliches) Ziel einer positiven Einwirkung auf Gesellschaft und Umwelt durch die Non-Profit-Organisation B Lab haben zertifizieren lassen.

Plattformen mit hybridem Geschäftsansatz können als For-Profit-Unternehmen zum Versprechen der Unabhängigkeit beitragen. Allerdings gelten auch hier die im Zusammenhang mit der Gewinnorientierung diskutierten Einschränkungen durch eine mögliche Transaktionsgebühr. Hinsichtlich des Nachhaltigkeitsversprechens besitzt die zu profitgetriebenen Plattformen geführte Diskussion für hybride Plattformen ebenfalls Gültigkeit, weil sich auch in ihrem Fall Anreize für das Einführen zusätzlicher Ressourcen herausbilden können. Bezüglich des Demokratisierungsversprechens stellt sich die Frage, ob eine Zertifizierung als B Corp bereits ausreicht, um eine Community von Gleichgesinnten 
anzuziehen. Diese Frage kann letztlich nur empirisch beantwortet werden, aber der Blick auf die von Muñoz und Cohen für diesen Geschäftsansatz angeführten Beispiele Kickstarter und Etsy lässt vermuten, dass hier keine nennenswerten positiven Auswirkungen zu erwarten sind.

\subsection{Tauschobjekt}

In der Dimension Tauschobjekt (»shared resources«) berufen sich Muñoz und Cohen (implizit) auf zwei Kriterien, um Plattformtypen zu unterscheiden. Zum einen berücksichtigen sie die Eigentumsverhältnisse: Die Tauschobjekte können entweder nach der Transaktion im Besitz der Anbietenden bleiben (Verleih, Vermietung) oder aber in den Besitz der Nachfragenden übergehen (Verkauf, Schenkung). Muñoz und Cohen gehen also, wie der Großteil der Literatur, von einem weit gefassten Sharing-Begriff aus, der eine ganze Reihe an Aktivitäten enthält: »traditional sharing, bartering, lending, trading, renting, gifting and swapping « (Botsman und Rogers 2011, xv). Zum anderen berücksichtigen sie den Zeitraum, in dem sich das Tauschobjekt im Besitz der Anbietenden befindet: Sie können dieses zum Beispiel bereits länger besitzen, eventuell ohne es bislang auf einer Sharing-Plattform angeboten zu haben. In diesem Fall ist davon auszugehen, dass dieses ursprünglich aufgrund seines Nutzwerts angeschafft wurde, wie etwa die Bohrmaschine, die nach Erfüllung ihres Zwecks ungenutzt im Regal liegt. Demgegenüber können Anbietende das Tauschobjekt aber auch genau mit dem Ziel angeschafft haben, es auf einer Sharing-Plattform anzubieten. In diesem Fall ist der Tauschwert des Objekts für die Anschaffung ausschlaggebend. Mit Bezug auf diese beiden Kriterien leiten

Tab. 5: Auswirkungen der Ausprägungen in der Dimension Tauschobjekt auf die Versprechen der Sharing Economy (Legende siehe Tab. 1).

\begin{tabular}{|c|c|c|c|c|}
\hline & \multicolumn{4}{|c|}{ Versprechen } \\
\hline & \multirow{2}{*}{$\begin{array}{l}\text { Nachhal- } \\
\text { tigkeit }\end{array}$} & \multicolumn{2}{|l|}{ Demokratisierung } & \multirow{2}{*}{$\begin{array}{l}\text { Unabhän- } \\
\text { gigkeit }\end{array}$} \\
\hline & & Entscheidungsrechte & Sozialkapital & \\
\hline Unternutzte & $\boldsymbol{1}$ & $\emptyset$ & $\emptyset$ & $\boldsymbol{1}$ \\
\hline Optimierte neue & 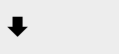 & $\emptyset$ & $\emptyset$ & $\boldsymbol{1}$ \\
\hline Besitzwechselnde & - & $\emptyset$ & $\emptyset$ & 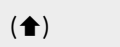 \\
\hline
\end{tabular}


Muñoz und Cohen dann drei Kategorien ab: unternutzte Tauschobjekte (»under-utilized«), optimierte neue Tauschobjekte (»optimize new«) und besitzwechselnde Tauschobjekte (»new home 4 used«). Tab. 5 liefert einen Überblick über deren Auswirkungen.

\section{Unternutzte Tauschobjekte}

Laut Muñoz und Cohen umfasst die Kategorie unternutzte Tauschobjekte solche Objekte, die sich bereits zuvor im Besitz der Anbietenden befanden und die nun zur zeitweiligen Nutzung auf einer SharingPlattform angeboten werden. Beispielhafte Plattformen für diese Kategorie sind Airbnb für Übernachtungsmöglichkeiten, Peerby für Haushaltsgegenstände und BlaBlaCar für Reisemöglichkeiten. Aus analytischer Perspektive sei hier angemerkt, dass die pauschale Zuordnung einer Plattform wie Airbnb zu dieser Kategorie nicht gerechtfertigt ist. Die Zuordnung sollte anstatt auf der Ebene der Plattform auf der Ebene der einzelnen Transaktionen stattfinden: Es macht einen Unterschied, ob das nach dem Auszug des Kindes freigewordene Gästezimmer auf der Plattform angeboten oder ob eine Wohnung eigens zur Untervermietung über Airbnb angemietet wird. Der zweite Fall ist analytisch der Kategorie optimierte neue Tauschobjekte zuzuordnen.

Plattformen der Kategorie unternutzte Tauschobjekte können zu einer nachhaltigeren Nutzung von Ressourcen beitragen. Sie verkörpern damit die ikonische Erzählung der gemeinsam genutzten Bohrmaschine. Bei diesem Plattformtyp zeigt sich besonders gut, wie die Sharing Economy dazu beitragen kann, eine generellere gesellschaftliche Verschiebung der Prioritäten von Besitz zum Zugang zu Objekten (Rifkin 2000; Belk 2007) zu unterstützen: Teilen ist das neue Besitzen.

Auch ermöglichen es Plattformen dieser Kategorie - in Kombination mit einem entsprechenden Transaktionstyp - ihren Nutzer*innen, neue Einkommensquellen zu erschließen. Im Endeffekt besteht das Prinzip bei all solchen Plattformen, auf denen physische Objekte getauscht werden, ${ }^{3}$ daraus, dass Kapital eingesetzt wird, um eine Rendite zu erwirtschaften. Als Kapital dienen bei Plattformen der Kategorie unternutzte Tauschobjekte bislang nicht als produktives Kapital behandelte Objekte

3 Zur Unterscheidung zwischen den physischen Tauschobjekten sowie Zeit und Fähigkeiten als Tauschobjekten siehe Frenken (2017). Allerdings trifft Frenken die nicht näher begründete und zumindest ungewöhnliche Entscheidung, Zeit und Fähigkeiten als Tauschobjekte definitorisch aus der Sharing Economy auszuschließen. 
im Besitz der Anbietenden. Das Auftreten als Anbietende setzt damit allerdings voraus, dass man überhaupt über solches Kapital verfügt. Fehlt dieses dagegen, bleibt auch das Versprechen auf Unabhängigkeit ein leeres. Anders verhält es sich bei Plattformen, bei denen keine physischen Objekte getauscht werden, sondern Zeit samt Fähigkeiten als Tauschobjekt fungieren. Das ist etwa bei Zeitbanken wie TimeRepublik der Fall, aber auch bei Service-Plattformen wie TaskRabbit für haushaltsnahe Dienstleistungen oder Helpling speziell für häusliche Reinigungsarbeiten. Hier wird kein Kapital investiert, sondern Arbeitskraft verkauft. Fungiert die Arbeitskraft als Tauschobjekt, ist die Anbietendenposition, zumindest in der Theorie, deutlich inklusiver angelegt, da wesentlich mehr Menschen in der Lage sein dürften, ihre Arbeitskraft zu verkaufen als Kapital zu investieren.

\section{Optimierte neue Tauschobjekte}

Auf Plattformen, die zur Kategorie optimierte neue Tauschobjekte gehören, werden Objekte zum Tausch gebracht, die Anbietende eigens zum Zweck des Anbietens auf diesen Plattformen angeschafft haben. Darunter fallen beispielsweise die Fahrzeugvermietung Share Now, aber eben auch bestimmte Transaktionen »gemischter « Plattformen wie Airbnb.

Ihr Transaktionsprinzip gleicht denen der Kategorie unternutzte Tauschobjekte. Allerdings wirkt sich die Neuanschaffung darauf aus, wie gut diese Plattformen die Versprechen der Sharing Economy erfüllen können. Im Gegensatz zur ersten Kategorie werden nämlich zusätzliche Ressourcen verbraucht, um die neu anzuschaffenden Objekte zu produzieren: Share Now vermietet Neuwagen statt bereits genutzte Fahrzeuge. Auch wenn diese Wagen intensiver genutzt werden sollten als Privatfahrzeuge, führen Plattformen dieses Typs daher zwangsläufig zu einem zusätzlichen Ressourcenverbrauch. Entsprechend ist ihr Beitrag zu einem nachhaltigen Wirtschaften als deutlich geringer einzuschätzen.

Ähnlich wie Plattformen der Kategorie unternutzte Tauschobjekte ermöglichen es Plattformen der Kategorie optimierte neue Tauschobjekte ihren Nutzer*innen wiederum, neue Einkommensquellen zu erschließen. Im Vergleich zur ersten Kategorie spitzt sich hier allerdings die Frage des Kapitalbesitzes als Zugangsvoraussetzung zur Anbietendenposition zu. So mag es sein, dass man ein Auto besitzt, das man in den ungenutzten Zeiten anderen über eine Plattform wie SnappCar überlässt und durch diesen Kapitaleinsatz eine Rendite erwirtschaftet. Die 
Anschaffung eines Wagens hingegen, der nicht für den Eigenbedarf genutzt werden soll, sondern eine reine Investition darstellt, erfordert das Vorhandensein eines zu Anlagezwecken einsetzbaren Kapitals.

\section{Besitzwechselnde Tauschobjekte}

Während die Tauschobjekte bei Plattformen der ersten beiden Typen im Besitz der Anbietenden verbleiben, gehen sie bei Plattformen des besitzwechselnden Typs in den Besitz der Nachfragenden über. Das ist etwa bei Versteigerungsplattformen wie eBay der Fall, aber auch bei Tauschplattformen wie Tauschgarten. Anbieter wie Kleiderkreisel erlauben sowohl Tausch als auch Verkauf. Ähnlich wie Airbnb bei der kurzfristigen Überlassung operiert jedoch auch eBay mit einem hybriden Ansatz und lässt den Verkauf gebrauchter wie neuer Waren zu. Für den zweiten Fall, den Verkauf oder Tausch eigens für diese Transaktion über die Plattform erworbener Objekte, sieht der Kompass von Muñoz und Cohen allerdings keine eigene Kategorie vor.

Hinsichtlich des Versprechens auf Nachhaltigkeit lässt sich für Plattformen dieses dritten Typs ein positiver Einfluss konstatieren. Indem nicht mehr genutzte Objekte über die Plattform feilgeboten werden und in den Besitz von Nutzer*innen gelangen, die ihnen einen Gebrauchswert beimessen, wird ihre Nutzungsdauer verlängert. Gleichzeitig müssen Nachfragende keine neu produzierten Objekte erwerben. Diese Einschätzung gilt selbstverständlich nur für den von Muñoz und Cohen vorgesehenen Fall der bereits gebrauchten Tauschobjekte. Das Versprechen auf Unabhängigkeit können Plattformen dieses Typs mit einem entsprechenden Transaktionsmodell ebenfalls unterstützen, indem sie nicht gebundenes, da nicht mehr genutztes Warenkapital durch Verkauf oder Tausch in Geldkapital verwandeln. Allerdings sind diesem Vorgang engere Grenzen gesetzt als in den Fällen der ersten beiden Plattformtypen: Ein Tauschobjekt kann nur einmal verkauft oder getauscht werden, während es beliebig oft verliehen oder vermietet werden kann.

\subsection{Technologie}

Mit der Dimension Technologie erfassen Muñoz und Cohen (2018, 130) den Stellenwert, den digitale Technologien für eine Sharing-Plattform besitzen: „We define technology as the reliance on digital technologies for facilitating discovery and exchange on the platform.«Der Kompass 
unterscheidet drei Grade: niedriger/kein Technologieeinsatz (»low/notech«), durch Technologie ermöglicht (»tech-enabled«) und technologiebetrieben (»tech-driven«). Allerdings ist diese Konzeption nur bedingt sinnvoll: Erstens scheint bei Plattformen die Möglichkeit einer Technologielosigkeit (»no-tech«) ausgeschlossen. Zweitens wirkt die von Muñoz und Cohen als Nebenkriterium für Plattformen mit niedrigem/ keinem Technologieeinsatz angeführte gemeinsame Nutzung einer physischen Infrastruktur wenig überzeugend. Denn auch bei Uber, das nach Muñoz und Cohen zu den technologienutzenden Plattformen gehört, wird der Zugang zu einem physischen Gut in Form des Fahrzeugs ermöglicht. Es liegt daher nahe, vorzuschlagen, die Unterscheidung vorranging auf den Einsatz einer technologischen Lösung zur Schaffung eines Marktes zu beziehen; hier zeigt sich eben im Fall von Uber, wie sehr die Transaktionen auf vom Algorithmus durchgeführten Operationen beruhen. Dieser berechnet, welche Nachfragenden mit welchen Anbietenden zu welchem Preis und zu welcher Zeit zusammengebracht werden.

Drittens, und das ist unser Haupteinwand, sind die beiden letzten Kategorien nicht trennscharf. Muñoz und Cohens Erklärung beschränkt sich auf den Hinweis, dass Plattformen der ersten Kategorie von Faceto-face-Interaktionen "profitieren « (ebd., 130). Weil die Autoren aber kein Beispiel für die erste Kategorie benennen und einzig Uber als Beispiel für die zweite Kategorie anführen, es aber auch bei Uber-Transaktionen in Zeiten nicht-autonomen Fahrens zwangsläufig zu Face-toface-Interaktionen kommt, sind diese Interaktionen kein geeignetes Unterscheidungskriterium. So scheint sich die Dreiteilung vielmehr aus dem Wunsch nach Symmetrie denn aus inhaltlichen Gründen zu ergeben. Wir schlagen daher vor, lediglich zwei Ausprägungen zu unterscheiden: Low Tech und High Tech (siehe Tab. 6).

Tab. 6: Auswirkungen der Ausprägungen in der Dimension Technologie auf die Versprechen der Sharing Economy (Legende siehe Tab. 1).

\begin{tabular}{|c|c|c|c|c|}
\hline & \multicolumn{4}{|c|}{ Versprechen } \\
\hline & \multirow{2}{*}{$\begin{array}{l}\text { Nachhal- } \\
\text { tigkeit }\end{array}$} & \multicolumn{2}{|l|}{ Demokratisierung } & \multirow{2}{*}{$\begin{array}{l}\text { Unabhän- } \\
\text { gigkeit }\end{array}$} \\
\hline & & Entscheidungsrechte & Sozialkapital & \\
\hline Low Tech & $(\mathrm{p} 2 \mathrm{p})$ & $\boldsymbol{F}(\mathrm{p} 2 \mathrm{p})$ & $(\mathrm{p} 2 \mathrm{p})$ & (p2p) \\
\hline High Tech & - $(\mathrm{p} 2 \mathrm{p})$ & - $(p 2 p)$ & - $(p 2 p)$ & - $(\mathrm{p} 2 \mathrm{p})$ \\
\hline
\end{tabular}


Low Tech

Für den Typ Low Tech lässt sich feststellen, dass der weitgehende Verzicht auf eine technologische Lösung bei der Schaffung eines Marktes die Plattformen eines grundlegenden Vorteils beraubt, der der Sharing Economy überhaupt erst ihren Status als potenzielle Transformationsagentin verleiht. Denn es ist die Netzwerkarchitektur des Internets und damit die technologische Basis, die es erleichtert, schwache Verbindungen (sensu Granovetter 1973) zwischen Peers herzustellen, indem sie lokale, temporale und soziale Beschränkungen entscheidend verringert. Entsprechend gilt für Plattformen dieses Typs, dass sie nicht in vergleichbarem Ausmaß zur Entstehung von p2p-Beziehungen beitragen können, womit ihr Beitrag zur Realisierung der drei Versprechen als gering einzuschätzen ist.

\section{High Tech}

Plattformen der Kategorie High Tech nutzen digitale Technologien, um einen Markt zu schaffen, und sind dadurch in der Lage, die Beschränkungen bei der Etablierung schwacher Verbindungen zwischen Marktteilnehmenden zu verringern. Auf diese Weise können sie, einen entsprechenden Plattformtyp vorausgesetzt, zur Entstehung von p2p-Transaktionen beitragen und so indirekt die Realisierung der Versprechen unterstützen.

\section{Eine transformative Sharing Economy ist möglich}

Die Diskussion der Dimensionen des »Sharing business model compass« von Muñoz und Cohen (2018) hat gezeigt, wie sich die Entscheidung der Betreiber*innen für ein bestimmtes Geschäftsmodell auf das Potenzial ihrer Plattform - und damit, in Summe, der Sharing Economy - auswirkt, eine Große Transformation zu unterstützen. Für jede Dimension wurde der jeweils mögliche Beitrag seiner Ausprägungen zur Erreichung dieses Ziels herausgearbeitet.

Die vollständige Beschreibung eines Geschäftsmodells erhält man jedoch erst, wenn man alle sechs Kompassdimensionen in ihrer Kombination betrachtet. Auf Grundlage unserer Analyse lässt sich nun bestimmen, welches Zusammenspiel von Ausprägungen besser oder weniger gut geeignet ist, um eine Transformation zu unterstützen. Tab. 7 stellt die günstigste und ungünstigste Kombination vor. 
Tab. 7: Am ehesten für eine Transformation geeignete und am wenigsten geeignete Kombination von Ausprägungen der Geschäftsmodelldimensionen von Sharing-Plattformen.

\begin{tabular}{|l|l|l|}
\hline & $\begin{array}{l}\text { geeignetste } \\
\text { Ausprägungskombination }\end{array}$ & $\begin{array}{l}\text { ungeeignetste } \\
\text { Ausprägungskombination }\end{array}$ \\
\hline Plattform & $\mathrm{p} 2 \mathrm{p}$ & b2c/b2b \\
\hline Transaktion & $\begin{array}{l}\text { Abhängig von Priorisierung der } \\
\text { Versprechen }\end{array}$ & $\begin{array}{l}\text { Abhängig von Priorisierung der } \\
\text { Versprechen }\end{array}$ \\
\hline Governance & $\begin{array}{l}\text { Kooperativ (Variante 2: } \\
\text { \#pen Cooperativism «) }\end{array}$ & Korporativ/Kollaborativ \\
\hline Geschäftsansatz & Missionsgetrieben & Profitgetrieben \\
\hline Tauschobjekt & Unternutzte T0 & Optimierte neue Tauschobjekte \\
\hline Technologie & High Tech & Low Tech \\
\hline
\end{tabular}

Der Blick auf die Flaggschiffe der Sharing Economy - dazu zählen unter anderem Airbnb, BlablaCar, eBay, TaskRabbit und Uber - offenbart nun allerdings, dass ihre Geschäftsmodelle tendenziell nahe an der ungünstigsten Kombination liegen. Tab. 8 illustriert dies exemplarisch an den Plattformen Uber sowie Airbnb und schlüsselt insbesondere den auf Uber dominanten und auf Airbnb im Aufstieg begriffenen (Twickel 2020) Fall der professionellen Anbietenden gesondert auf.

Tab. 8: Transformatives Potenzial der Geschäftsmodelle von Uber und Airbnb.

\begin{tabular}{|l|l|l|l|l|}
\hline & $\begin{array}{l}\text { Uber: } \\
\text { professionelle Fahrer*innen } \\
\text { Airbnb: } \\
\text { professionelle Vermieter*innen }\end{array}$ & $\begin{array}{l}\text { Uber: } \\
\text { gelegentliche Fahrer*innen } \\
\text { Airbnb: } \\
\text { gelegentliche Vermieter*innen }\end{array}$ \\
\hline Plattform & b2c & & p2p & \\
\hline Transaktion & Markt & $\emptyset$ & Markt & $\emptyset$ \\
\hline Governance & Korporativ & - & Korporativ & \\
\hline Geschäftsansatz & Profitgetrieben & - & Profitgetrieben & \\
\hline Tauschobjekt & Optimierte neue T0 & - & Unternutzte T0 & \\
\hline Technologie & High Tech & - & High Tech & \\
\hline
\end{tabular}


Bei diesen Flaggschiffen handelt es sich durchweg um Plattformen, die nach dem oben eingeführten Raster von Hertwig und Papsdorf (2017) dem Segment der Plattformökonomie zugehören. Auch im Hinblick auf die Geschäftsmodelle gilt also, was sich bereits in der diskursiven Legitimierung der Sharing Economy beobachten ließ (Jürss und Borchers 2020): Das in der real existierenden Sharing Economy dominierende Segment der Plattformökonomie rechtfertigt nicht die in die Sharing Economy gesetzten Hoffnungen. Diese Hoffnungen beruhen vielmehr auf anderen, nicht der Plattformökonomie zuzuordnenden Plattformen wie Tauschgarten oder foodsharing, deren wünschenswerte Auswirkungen durch einen ideologisch motivierten »Brückenschlag« (ebd., 194) auch für die Plattformökonomie in Anspruch genommen werden.

Ungeachtet dieser Beschränkungen der real existierenden Sharing Economy legt unsere Analyse jedoch nahe, dass Sharing-Plattformen, ein entsprechendes Geschäftsmodell vorausgesetzt, durchaus einen Beitrag zu einer sozial-ökologischen Transformation leisten könnten. Eine besondere Aufgabe kommt hier Plattformen zu, die das von uns als am günstigsten identifizierte Geschäftsmodell - sozusagen den Idealtyp einer transformativen Sharing-Plattform - adaptieren. Sie können einen Baustein in der »realen Utopie« (Wright 2017) einer nachkapitalistischen Gesellschaft bilden. Andere Plattformen, die eine Variation dieses Geschäftsmodells adaptieren, sind in diesem Zusammenhang ebenfalls von Hilfe. Entsprechende Abstufungen lassen sich auf Grundlage einer Priorisierung der Versprechen entwickeln. Gewichtet man etwa das Versprechen des nachhaltigen Wirtschaftens höher als das Versprechen, neue, unabhängige Einkommensquellen zu erschließen eine Entscheidung, die etwa aus einer ökosozialistischen Perspektive (Kovel 2007) notwendig erscheint -, sind der freie und der alternative Transaktionstyp dem markt-förmigen vorzuziehen.

Um das transformative Potenzial der Sharing Economy nutzbar zu machen, müssten also gerade solche Plattformen eine größere Rolle im Feld spielen, deren Geschäftsmodell sich am Idealtyp einer transformativen Plattform orientiert. Zwar sind uns keine Anbieter bekannt, die diesen Idealtyp in Reinform verkörpern, es gibt allerdings Plattformen, die sich ihm bereits annähern wie etwa foodsharing, Tauschgarten oder BeWelcome. Es ist also notwendig, Projekte dieser Art gezielt aufzubauen und ihre Nutzung zu popularisieren. Denn es dürfte klar sein, dass sie aus der Perspektive von Investor*innen, die den Imperativen eines kapitalistischen Wirtschaftssystems folgen, deutlich weniger an- 
sprechend erscheinen als Plattformen des von uns als am ungünstigsten identifizierten Typs. Die geringere Attraktivität für Investor*innen zeigt sich besonders offensichtlich an der Adaption eines missions- statt eines profitgetriebenen Geschäftsansatzes, aber auch in anderen Dimensionen des Geschäftsmodells wie dem Verzicht auf Zulassung von b2c-Transaktionen und von neuen Tauschobjekten sowie an der Wahl eines kooperativen statt korporativen Governance-Typs.

Um transformationsunterstützende Sharing-Plattformen auch ohne die Dynamiken eines kapitalistischen Marktes etablieren zu können, ist daher zum einen die Arbeit von Aktivist*innen nötig, die bereit sind, missionsgetriebene Plattformen aufzusetzen und am Leben zu halten. Zum anderen sind die Nutzer*innen gefragt, sich im Rahmen von Plattformkooperativen einzubringen. Weil die Transformation schließlich nicht einzig von Individuen geschultert werden kann, ist zudem eine politische Unterstützung entsprechender Plattformen notwendig. Diese Unterstützung sollte von finanziellen Hilfen bis hin zu Veränderungen der »Marktregeln« zugunsten transformationsunterstützender Plattformen, die etwa den Nachteil begrenzter UpscalingMöglichkeiten ausgleichen, reichen. Beispielweise ließe sich nachdenken über nichtkommerzielle lokale Sharing-Gemeinschaften für Gegenstände des alltäglichen und nicht alltäglichen Bedarfs (Rasenmäher, Festzelte, Campingausrüstung), die auf einer gemeinsamen, von Städten und Gemeinden finanzierten technischen Plattform aufbauen. Überlegenswert ist es ebenfalls, Marktbedingungen zugunsten genossenschaftlich organisierter Plattformen im Sinne von Felbers Gemeinwohl-Ökonomie (2014) zu verändern, etwa durch Steuervergünstigungen oder die bevorzugte Vergabe von Aufträgen oder Privilegien. Beispielsweise könnten Städte und Gemeinden genossenschaftlich organisierten »Mobilitätsunternehmen« (Kurzzeitleihe von Fahrrädern, E-Rollern, Autos und anderem) bevorzugt günstige Park- und Abstellpunkte zuweisen.

\section{Literatur}

Acquier, Aurélien, Thibault Daudigeos, und Jonatan Pinkse. 2017. Promises and paradoxes of the sharing economy: An organizing framework. Technological Forecasting and Social Change 125: 1-10. DOI: https://doi.org/10.1016/j.techfore.2017.07.006.

Bauwens, Michel, und Vasilis Kostakis. 2014. From the communism of capital to capital for the commons: Towards an open co-operativism. TripleC: Communica- 
tion, Capitalism \& Critique 12 (1): 356-361. http://www.triple-c.at/index.php/ tripleC/article/view/561/678.

Belk, Russell. 2007. Why not share rather than own? The ANNALS of the American Academy of Political and Social Science 611 (1): 126-140. DOI: https://doi. org/10.1177/0002716206298483.

Borchers, Nils S., und Nicola Krömer. 2015. Die Rolle von Arztbewertungsportalen im Prozess der Arztwahl: Patienten-Empowerment durch Laienbewertungen? In: Gesundheitskommunikation im gesellschaftlichen Wandel, herausgegeben von Markus Schäfer, Oliver Quiring, Constanze Rossmann, Matthias R. Hastall und Eva Baumann, 153-164. Baden-Baden: Nomos.

Botsman, Rachel, und Roo Rogers. 2011. What's mine is yours: How collaborative consumption is changing the way we live. London: Collins.

Brecht, Bertolt. 1982 [1932/33]. Der Rundfunk als Kommunikationsapparat. In: Gesammelte Werke in 20 Bänden, Band 18, 127-134. Frankfurt am Main: Suhrkamp.

Buchstein, Hubertus. 1997. Bytes that bite: The internet and deliberative democracy. Constellations 4 (2): 248-263. DOI: https://doi.org/10.1111/14678675.00052 .

Chase, Robin. 2015. Peers Inc: How people and platforms are inventing the collaborative economy and reinventing capitalism.

Daum, Timo. 2017. Das Kapital sind wir: Zur Kritik der digitalen Ökonomie. Hamburg: Nautilus.

Decrop, Alain, Giacomo Del Chiappa, Jérôme Mallargé und Pietro Zidda. 2018. "Couchsurfing has made me a better person and the world a better place": The transformative power of collaborative tourism experiences. Journal of Travel \& Tourism Marketing 35 (1): 57-72. DOI: https://doi.org/10.1080/10548408.201 7.1307159 .

Elias, Norbert. 2009 [1970]. Was ist Soziologie? 11. Auflage. Weinheim: Juventa.

Enzensberger, Hans Magnus. 1997 [1970]. Baukasten zu einer Theorie der Medien: Kritische Diskurse zur Pressefreiheit. München: Fischer.

Felber, Christian (2014). Die Gemeinwohl-Ökonomie: Eine demokratische Alternative wächst. Aktualisierte und erweiterte Neuausgabe. Wien: Deuticke.

Frenken, Koen. 2017. Political economies and environmental futures for the sharing economy. Philosophical Transactions of the Royal Society A: Mathematical, Physical \& Engineering Sciences 375 (2095): 1-15.

Gansky, Lisa. 2010. The mesh: Why the future of business is sharing. New York: Penguin.

Göpel, Maja. 2020. Unsere Welt neu denken: Eine Einladung. Berlin: Ullstein.

Granovetter, Mark S. 1973. The strength of weak ties. American Journal of Sociology 78 (6): 1360-1380.

Hamari, Juho, Mimmi Sjöklint, und Antti Ukkonen. 2015. The sharing economy: Why people participate in collaborative consumption. Journal of the Association for Information Science and Technology. DOI: https://doi.org/10.1002/ asi.23552.

Hertwig, Markus, und Christian Papsdorf. 2017. Varieties of Sharing: Handlungs- 
orientierungen, Strukturen und Arbeitsbedingungen eines neuartigen Feldes. Berliner Journal für Soziologie 27 (3-4): 521-546. DOI: https://doi.org/10.1007/ s11609-018-0359-5.

Hill, Steven. 2015. Raw deal: How the »Uber economy « and runaway capitalism are screwing American workers. New York: St. Martin's.

Jürss, Sebastian, und Nils S. Borchers. 2020. Die Ideologie der Sharing Economy. In: Ideologie, Kritik, Öffentlichkeit: Verhandlungen des Netzwerks Kritische Kommunikationswissenschaft, herausgegeben von Uwe Krüger und Sebastian Sevignani, 188-211. Frankfurt am Main: Westend. DOI: https://doi. org/10.36730/ideologiekritik.2019.9.

Kearney. (о. J.). The demystification of car sharing: An in-depth analysis of customer perspective, underlying economics, and secondary effects. https://www.de.kearney.com/automotive/article?/a/the-demystification-of-car-sharing. Zugegriffen: 1. Mai 2021.

Kovel, Joel. 2007. The enemy of nature: The end of capitalism or the end of the world? 2. Auflage. London: Zed.

Labrecque, Lauren I., Jonas vor dem Esche, Charla Mathwick, Thomas P. Novak, und Charles F. Hofacker. 2013. Consumer power: Evolution in the digital age. Journal of Interactive Marketing 27 (4): 257-269. DOI: https://doi.org/ 10.1016/j.intmar.2013.09.002.

Lessig, Lawrence. 2006. Code: Version 2.0. 2. Auflage. New York: Basic.

Liu, Yaping, Linlin Nie, und Li Li. 2016. Homogeneity, trust, and reciprocity: Three keys to the sustainable hospitality exchange of Couchsurfing. Tourism Analysis 21 (2): 145-157. DOI: https://doi.org/10.3727/108354216X14559233984610.

Mikołajewska-Zaj c, Karolina. 2016. Sharing as labour and as gift: Couchsurfing as an »affective enterprise«. Ephemera 16 (4): 209-222.

Muñoz, Pablo und Boyd Cohen. 2017. Mapping out the sharing economy: A configurational approach to sharing business modeling. Technological Forecasting and Social Change 61 (1): 114-147. DOI: https://doi.org/10.1016/j.techfore.2017.03.035.

Muñoz, Pablo und Boyd Cohen. 2018. A compass for navigating sharing economy business models. California Management Review 61 (1): 114-147. DOI: https:// doi.org/10.1177/0008125618795490.

Murillo, David, Heloise Buckland und Esther Val. 2017. When the sharing economy becomes neoliberalism on steroids: Unravelling the controversies. Technological Forecasting and Social Change 125: 66-76. DOI: https://doi.org/10.1016/j.techfore.2017.05.024.

Pazaitis, Alex, Vasilis Kostakis und Michel Bauwens. 2017. Digital economy and the rise of open cooperativism: The case of the Enspiral Network. Transfer: European Review of Labour and Research 23(2): 177-192. DOI: https://doi. org/10.1177/1024258916683865.

Rifkin, Jeremy. 2000. The age of access: The new culture of hypercapitalism, where all of life is a paid-for experience. New York: Tarcher/Putnam.

Rifkin, Jeremy. 2014. The zero marginal cost society: The internet of things, the collaborative commons, and the eclipse of capitalism. New York: Palgrave Macmillan. 
Scholz, Trebor. 2017. Uberworked and underpaid: How workers are disrupting the digital economy. Cambridge: Polity.

Scholz, Trebor, und Nathan Schneider (Hrsg.). 2017. Ours to hack and to own: The rise of platform cooperativism, a new vision for the future of work and a fairer internet. New York: OR.

Schor, Juliet B. 2014. Debating the sharing economy. http://www.greattransition. org/publication/debating-the-sharing-economy. Zugegriffen: 3. März 2021.

Slee, Tom. 2015. What's yours is mine: Against the sharing economy. New York: OR. Srnicek, Nick. 2017. Platform Capitalism. Cambridge: Polity.

Twickel, Christoph. 2020. Mehr als Luftmatratze mit Frühstück. Die Zeit vom 30. November, https://www.zeit.de/wirtschaft/unternehmen/2020-11/airbnb-corona-wirtschaftskrise-boersengang-erfolg-unternehmen. Zugegriffen: 3. März 2021.

United Nations. o. J. 17 goals to transform our world. https://www.un.org/sustainabledevelopment/. Zugegriffen: 3. März 2021.

Wright, Erik Olin. 2017. Reale Utopien: Wege aus dem Kapitalismus. Frankfurt am Main: Suhrkamp.

\section{Open Access}

Dieser Beitrag erscheint unter der Creative-Commons-Lizenz CC BY-ND 3.0 DE: https://creativecommons.org/licenses/by-nd/3.0/de/. 